\title{
Ray tracing of whistler-mode chorus elements: implications for generation mechanisms of rising and falling tone emissions
}

\author{
K. Yamaguchi ${ }^{1}$, T. Matsumuro ${ }^{1}$, Y. Omura ${ }^{1}$, and D. Nunn ${ }^{1,2}$ \\ ${ }^{1}$ Research Institute for Sustainable Humanosphere, Kyoto University, Kyoto, 611-0011, Japan \\ ${ }^{2}$ School of Electronics and Computer Science, Southampton University, Southampton, Hampshire, SO17 1BJ, UK
}

Correspondence to: D. Nunn (dn@ecs.soton.ac.uk)

Received: 26 October 2012 - Revised: 18 February 2013 - Accepted: 15 March 2013 - Published: 12 April 2013

\begin{abstract}
Using a well-established magnetospheric verylow-frequency (VLF) ray tracing method, in this work we trace the propagation of individual rising- and fallingfrequency elements of VLF chorus from their generation point in the equatorial region of the magnetosphere through to at least one reflection at the lower-hybrid resonance point. Unlike recent work by Bortnik and co-workers, whose emphasis was on demonstrating that magnetospheric hiss has its origins in chorus, we here track the motion in the equatorial plane of the whole chorus element, paying particular regard to movement across field lines, rotation, and compression or expansion of the wave pulse. With a generation point for rising chorus at the equator, it was found the element wave pulse remained largely field aligned in the generation region. However, for a falling tone generation point at $4000 \mathrm{~km}$ upstream from the equator, by the time the pulse crosses the equator the wavefield had substantial obliquity, displacement, and compression, which has substantial implications for the theory of falling chorus generation.
\end{abstract}

Keywords. Space Plasma Physics (Nonlinear phenomena; Wave-particle interactions; Waves and instabilities)

\section{Introduction}

Chorus is a radio science phenomenon that has long stimulated theoretical research, and with the advent of new and excellent satellite observations, such as from the CLUSTER and THEMIS satellites (Santolik et al., 2003; Li et al., 2011), it is once again a topic of great interest because of its role in energizing $\mathrm{MeV}$ electrons in the magnetosphere (Horne and Thorne, 2003; Katoh and Omura, 2007b) and also as an agency for precipitation of these energetic electrons into the ionosphere (Hikishima et al., 2010b). Chorus comprises self-sustaining very-low-frequency (VLF) radio emissions observed in the earth's magnetosphere, predominantly outside the plasmapause, and seen at all local times but with a pronounced minimum at dusk. Chorus divides into two categories - lower-band chorus in the band $0.2-0.5 \Omega_{\mathrm{e}} / 2 \pi$, where $\Omega_{\mathrm{e}} / 2 \pi$ is the local electron gyrofrequency, and upperband chorus in the band $0.5-0.7 \Omega_{\mathrm{e}} / 2 \pi$ (Hayakawa et al., 1984). Some recent observations, however, from THEMIS (Kurita et al., 2012) report individual elements spanning the frequency range $1-2.5 \mathrm{kHz}$ and completely crossing half the equatorial gyrofrequency. Chorus may be spectrally unstructured but more normally consists of series of risingfrequency or falling-frequency elements not unlike triggered emissions.

Satellite observations from CLUSTER (Santolik and Gurnett, 2003; Santolik et al., 2003) indicate that chorus is usually observed propagating away from the equator pointing to a generation region at or near the equator, though more rarely on CLUSTER chorus is seen propagating towards the equator (Pickett et al., 2004).

There is general agreement amongst theoreticians and simulationists that the underlying generation mechanism is the electron cyclotron resonance with the narrow band or band limited VLF wavefield with a $\boldsymbol{k}$ vector either parallel or quasi-parallel to the ambient magnetic field, the free energy driving the instability coming from the anisotropy of the energetic electron distribution function in the $\mathrm{keV}-\mathrm{MeV}$ range. Since chorus (and triggered VLF emissions for that matter) are obviously nonlinear phenomena, then resonant particle behaviour must also be nonlinear. In fact such nonlinearity takes the form of resonant particle trapping (Omura et al., 2008; Nunn et al., 1997). Since the ambient medium is 
inhomogeneous through the $z$ dependence of $B_{0}(z)$ and also through the sweeping frequency, electrons become phase locked at a phase angle dependent on the net inhomogeneity, and such particles undergo large changes in energy and magnetic moment resulting in an "electron hole" or "hill" coinciding with the location of the particle trap in velocity space. (Omura et al., 2008; Nunn et al., 1997).

Since resonant energies increase rapidly away from the equator and strong $B_{0}$ gradients away from the equator prevent nonlinear trapping, then the theory is in agreement with observations in predicting an equatorial or near-equatorial generation region.

Since current theory and simulations predict and assume parallel propagation in the generation region on the grounds that growth rates are maximum for $k \| B$, the next critical question is whether propagation is observed to in fact be parallel there. Observations (Santolik et al., 2009) do indicate that this is the case, though there seem to be exceptions. In this regard, a recent paper (Li et al., 2011) finds consistently high degrees of obliquity in the case of falling-frequency emissions, but near parallelism in the case of risers, which is a finding requiring further investigation.

The self-consistent nonlinear wave-particle interaction problem in a parabolic inhomogeneity is clearly quite complex, and to this effect much effort has been expended on numerical simulation. Omura and co-workers (Katoh and Omura, 2007a; Hikishima et al., 2009, 2010a, b; Omura et al., 2008, 2009) have developed numerically intensive broadband particle-in-cell (PIC) codes that have successfully simulated rising chorus starting from broadband noise, and also reproduced successive rising elements. The codes only produce risers for which the generating point is located at the equator and the nonlinear generation region itself extends some 1000s of kilometers downstream from there. This is in excellent agreement with CLUSTER observations.

A second approach is due to Nunn and co-workers and is a Vlasov hybrid simulation (VHS) narrow-band code with a processing bandwidth of $\sim 100 \mathrm{~Hz}$, originally designed for the triggered emission problem but applicable at least to individual chorus elements but not to the entire chorus problem (Nunn et al., 1997, 2009). In a more recent development, the code has been used to verify the theoretical result in Katoh and Omura (2007a) that the sweeping frequency is due entirely to the advective term, and it was shown that for a riser the negative nonlinear component of resonant particle current $J_{b}$ parallel to the wave $B$ field sets up the necessary negative frequency gradient to give a rising tone. (Omura and Nunn, 2011; Nunn and Omura, 2012).

The VHS code is able to reproduce risers, fallers, and hooks, but faller simulation is difficult and achieved by having large linear growth rates which drive the wave profile upstream, which is in the opposite direction to group velocity and along the magnetic field line. When a faller is triggered, the generating region is always upstream from the equator where the net inhomogeneity factor $S$ is positive, whereas
$S$ is negative downstream from the equator where the riser generating region is to be found.

Both PIC and VHS codes assume exact parallel propagation and permit long-range coherent wave-particle interactions across the whole simulation region, $\sim 8000 \mathrm{~km}$ long and crossing the equator. In Nunn and Omura (2012) it was argued that in view of the tendency of chorus waves to become more oblique as they propagate away from the generation region, it was unrealistic to assume such coherency. Accordingly, the simulation box was restricted to the region upstream from the equator where inhomogeneity is positive. Under these circumstances the code readily and consistently produced falling tones, and furthermore the current $J_{b}$ was positive, setting up a positive frequency gradient which gave a falling tone.

It is clear then that the assumption of exactly parallel propagation in theory and simulation is at odds with experimental reality and a more unified approach is needed in which propagation conditions are taken into account. Accordingly, in this work we ray trace, forward in time, individual chorus elements, starting from their generation point. We will be particularly interested in the extent to which the actual wave pulses undergo compression, rotation, and displacement from the field line in that region surrounding the equator where nonlinear wave-particle interaction may take place. The other key issue is the degree of obliquity which the $\boldsymbol{k}$ vectors acquire in propagating from their generation point and while they are still in the interaction zone.

\section{The ray tracing computations}

Ray tracing of VLF waves in the magnetosphere is a well-established investigative technique (Haselgrove, 1954; Kimura, 1966), and has been developed continuously ever since (Horne, 1989; Hiroyasu and Masashi, 1987). It has been employed more recently for three-dimensional ray tracing studies of VLF waves in the earth's magnetosphere (Chen et al., 2009). The basic ray tracing equations may be written in the form (Hiroyasu and Masashi, 1987)

$$
\begin{aligned}
& \frac{\mathrm{d} r}{\mathrm{~d} t}=\frac{1}{\mu^{2}}\left(\rho_{r}-\mu \frac{\partial \mu}{\partial \rho_{r}}\right), \\
& \frac{\mathrm{d} \theta}{\mathrm{d} t}=\frac{1}{r \mu^{2}}\left(\rho_{\theta}-\mu \frac{\partial \mu}{\partial \rho_{\theta}}\right), \\
& \frac{\mathrm{d} \phi}{\mathrm{d} t}=\frac{1}{r \mu^{2} \sin \theta}\left(\rho_{\phi}-\mu \frac{\partial \mu}{\partial \rho_{\phi}}\right), \\
& \frac{\mathrm{d} \rho_{r}}{\mathrm{~d} t}=\frac{1}{\mu} \frac{\partial \mu}{\partial r}+\rho_{\theta} \frac{\mathrm{d} \theta}{\mathrm{d} t}+\rho_{\phi} \frac{\mathrm{d} \phi}{\mathrm{d} t} \sin \theta, \\
& \frac{\mathrm{d} \rho_{\theta}}{\mathrm{d} t}=\frac{1}{r}\left(\frac{1}{\mu} \frac{\partial \mu}{\partial \theta}-\rho_{\theta} \frac{\mathrm{d} r}{\mathrm{~d} t}+r \rho_{\phi} \frac{\mathrm{d} \phi}{\mathrm{d} t} \cos \theta\right),
\end{aligned}
$$


and

$\frac{\mathrm{d} \rho_{\phi}}{\mathrm{d} t}=\frac{1}{r \sin \theta}\left(\frac{1}{\mu} \frac{\partial \mu}{\partial \phi}-\rho_{\phi} \frac{\mathrm{d} r}{\mathrm{~d} t} \sin \theta-r \rho_{\phi} \frac{\mathrm{d} \theta}{\mathrm{d} t} \cos \theta\right)$,

where $\{r, \theta, \varphi\}$ are the usual spherical coordinates and refractive index $\mu$ is given by the cold plasma dispersion relation of Stix,

$A \mu^{4}+B \mu^{2}+C=0$,

where the familiar coefficients $A, B, C$ are defined in Stix (1992). The vector $\left\{\rho_{r}, \rho_{\theta}, \rho_{\varphi}\right\}$ represents the components of the wave vector $\boldsymbol{k}$ in spherical coordinates. These calculations will employ the ray tracing code due to Kimura and Goto (2010).

For simplicity, we assume a dipole magnetic field model and confine ourselves to a two-dimensional simulation in the plane containing both magnetic poles and at a specific longitude. The cold plasma density model used is the diffusive equilibrium (DE) model and is illustrated in Fig. 1b (Richardson and Sittler, 1990; Persoon et al., 2009).

The DE profiles are evaluated along each individual field line as follows.

We first introduce the function geopotential height $z$.

$z=\frac{r_{0}}{r}\left(r-r_{0}\right)$

where $r_{0}$ is a reference radius distance, and also scale height $H_{i}$,

$H_{i}=\frac{k T_{i}}{M_{i} g\left(r_{0}\right)}$,

where $k, T_{i}, M_{i}$, and $g\left(R_{0}\right)$ are Boltzmann's constant, temperature and mass of the $i$ th ion, and gravity acceleration at $r_{0}$, respectively.

We assume that electron density at any altitude is determined by the charge balance with the positive ions. Defining

$N_{r}(z)=\left[\sum_{i} \eta_{i} \exp \left(-\frac{z}{H_{i}}\right)\right]^{\frac{1}{2}}$

where $\eta_{i}$ is the percentage of $i$ th ion, then, absolute electron density is expressed as

$N_{e}(z)=N_{\theta}\left[\sum_{i} \eta_{i} \exp \left(-\frac{z}{H_{i}}\right)\right]^{\frac{1}{2}}$

where $N_{\theta}$ is total ion density at the reference level. The relative density of the $i$ th ion with respect to electron density is given by

$Q_{i}(z)=\frac{\eta_{i} \exp \left(-\frac{z}{H_{i}}\right)}{N_{r}^{2}(z)}$,

and then the absolute ion density for species $i$ is calculated as (a) Overview

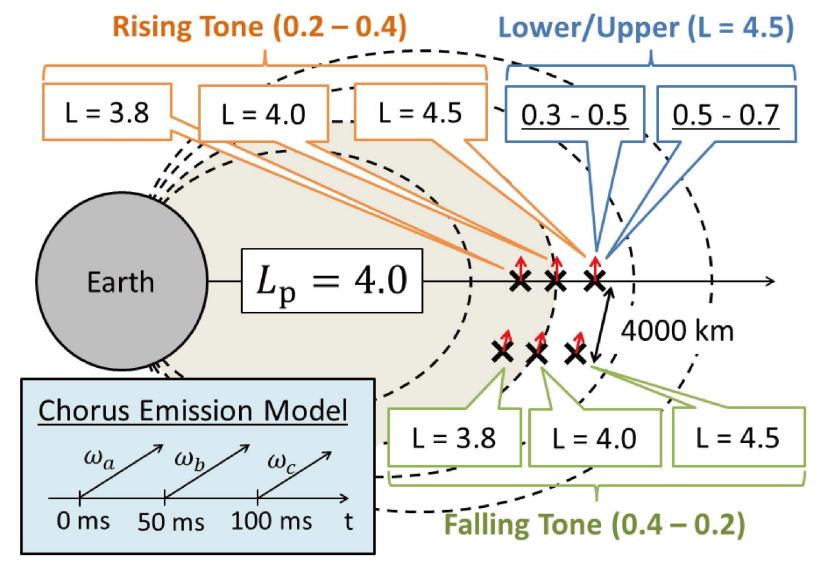

(b) Density model

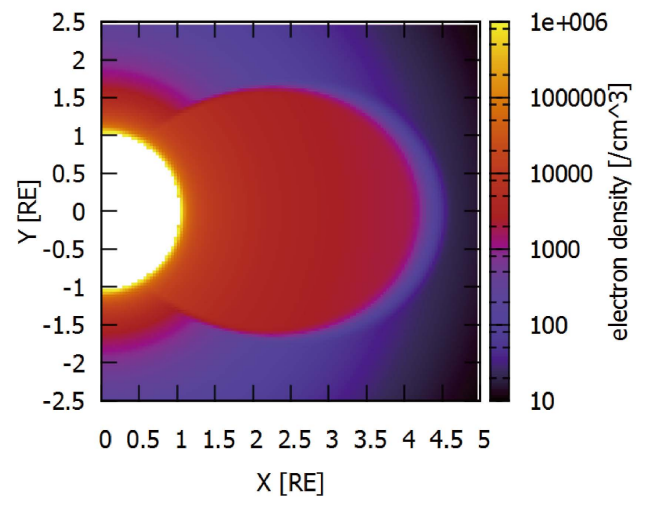

Fig. 1. (a) Meridional plane cross section of the magnetosphere indicating the plasmapause and the starting positions of the ray tracing. (b) Diffusive equilibrium model for cold plasma density.

$N_{i}=N_{e} Q_{i}$

Figure 1a gives a view of the fixed longitude plane showing plasmapause location at $L=4$ and indicating the starting points for the ray tracing of each chorus element. In accordance with the chorus equations (Omura et al., 2008, 2009), the generation point is at the magnetic equator for chorus rising elements, and the initial frequency sweeps linearly with time from $0.2-0.4 \Omega_{\mathrm{e}} / 2 \pi(\mathrm{Hz})$ in $50 \mathrm{~ms}$, where $\Omega_{\mathrm{e}} / 2 \pi$ is the local electron gyrofrequency. The elements are repeated every $50 \mathrm{~ms}$. The starting values for parameter $L$ are $L=3.8$ just inside the plasmapause, actually at the plasmapause $L=$ 4 , and outside the plasmapause $L=4.5$. While it is true that most chorus originates outside the plasmapause, the ray tracing starting from $L=3.8$ would be relevant to the closely allied problem of discrete and triggered VLF emissions. All ray tracing starts with zero propagation angle, or $\boldsymbol{k}$ vector parallel to $B_{0}(z)$, on the grounds that parallel propagation corresponds to maximum instability and observations (Santolik and Gurnett, 2003; Santolik et al., 2003, 2009) point to parallel or quasi-parallel propagation at the point of generation. 
Table 1. Latitude, distance from equator, local cyclotron and plasma frequencies at all the ray tracing start points.

\begin{tabular}{rrrrr}
\hline $\begin{array}{r}L \\
{[\mathrm{RE}]}\end{array}$ & $\begin{array}{r}\text { Latitude } \\
{[\mathrm{deg}]}\end{array}$ & $\begin{array}{r}\text { Distance from } \\
\text { equator }[\mathrm{km}]\end{array}$ & $\begin{array}{r}\text { Cyclotron } \\
\text { frequency }[\mathrm{kHz}]\end{array}$ & $\begin{array}{r}\text { Plasma frequency } \\
{[\mathrm{kHz}]}\end{array}$ \\
\hline 3.8 & 0 & 0 & 15.93 & 401.2 \\
3.8 & -9.5 & 4000 & 17.95 & 406.6 \\
4.0 & 0 & 0 & 13.66 & 391.3 \\
4.0 & -9.0 & 4000 & 15.26 & 396.1 \\
4.5 & 0 & 0 & 9.591 & 59.62 \\
4.5 & -8.0 & 4000 & 10.48 & 61.78 \\
\hline
\end{tabular}

However, in Fig. 6 the starting propagation angle will be the local Gendrin angle. The local values of electron plasma frequency, electron cyclotron frequency, latitude in degrees and distance from the equator are shown in Table 1.

The falling tone elements sweep linearly with time from $0.4-0.2 \Omega_{\mathrm{e}} / 2 \pi(\mathrm{Hz})$ in $50 \mathrm{~ms}$, the elements being repeated every $50 \mathrm{~ms}$. The starting points for the ray tracing are derived from the simulations in Nunn and Omura (2012), and in accordance with those results we take the generating point to be $4000 \mathrm{~km}$ upstream from the equator. The final exercise is a comparison between lower- and upper-band rising chorus, with element frequency ranges of $0.3-0.5 \Omega_{\mathrm{e}} / 2 \pi(\mathrm{Hz})$ and $0.5-0.7 \Omega_{\mathrm{e}} / 2 \pi(\mathrm{Hz})$, respectively, both extra-plasmapause at $L=4.5$.

In Fig. 2 we ray trace rising tone chorus elements $f=0.2$ $0.40 \Omega_{\mathrm{e}} / 2 \pi(\mathrm{Hz})$. Start points are with zero propagation angle at the equator at (a) $L=3.8$ (b) $L=4.0$ and (c) $L=4.5$. Left-hand panels show the physical extent and orientation in 2-D space of each rising frequency element wave pulse as time progresses. The wave pulse representations are coloured from red to blue to represent elapsed propagation time. For rising chorus the front of the pulse will have a frequency of $0.2 \Omega_{\mathrm{e}} / 2 \pi(\mathrm{Hz})$, and the rear of the pulse $0.40 \Omega_{\mathrm{e}} / 2 \pi$ $(\mathrm{Hz})$. The right-hand panels ray trace three rays at frequencies $f=0.2,0.3,0.4 \Omega_{\mathrm{e}} / 2 \pi(\mathrm{Hz})$ and show evolution of the $\boldsymbol{k}$ vector orientation with respect to $B_{0}$ field. All three starting points show common features, namely inward drift into the plasmasphere, the outermost ray at $L=4.5$ readily crossing the plasmapause. There is a very marked elongation of the wave pulses and rotation with respect to the magnetic field line so the pulse spans almost a whole $L$ value. By the time the wave reaches about 20 degrees of latitude the wave is very oblique and then remains so, close to the resonance cone. After lower-hybrid resonance reflection, there is no sign of any tendency towards parallel propagation when recrossing the equator as noted in Bortnik et al. (2011), though in that paper large numbers of rays were followed, including a whole range of starting propagation angles. This result is in agreement with a recent study (Breuillard et al., 2012) that employs Ronnmark's WHAMP hot plasma dispersion relation to ray trace large numbers of chorus rays originating at the equator from $L=4.5-7$. They found the propagation angle distribution at high latitudes to be very oblique and concentrated inside the local resonance cone. (a)
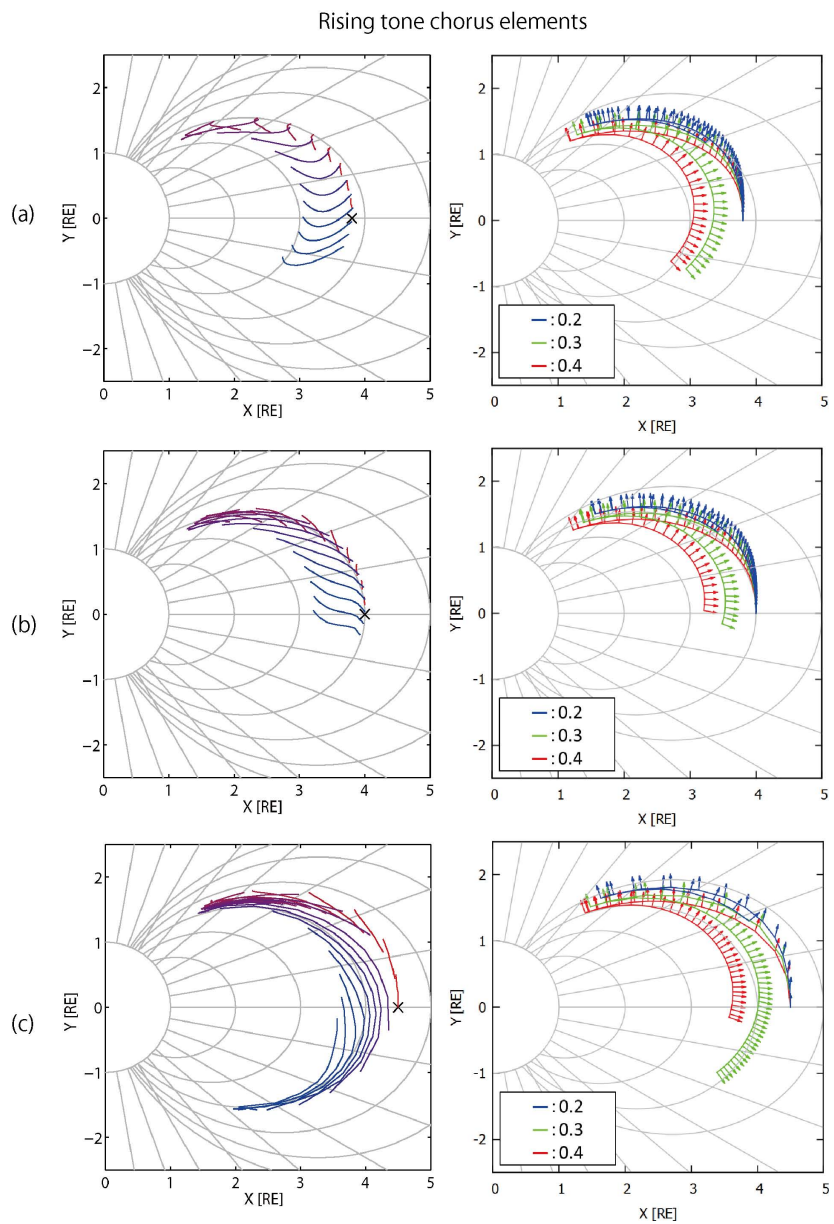

Fig. 2. Ray tracing of rising tone chorus elements with frequency span $f=0.2-0.40 \Omega_{\mathrm{e}} / 2 \pi(\mathrm{Hz})$, pulse length $50 \mathrm{~ms}$. Start points are with zero propagation angle at the equator and at (a) $L=3.8$ (b) $L=4.0$ and (c) $L=4.5$. Left-hand panels show the physical extent and orientation in 2-D space of each rising frequency element as time progresses. The right-hand panels ray trace three rays at frequencies $f=0.2,0.3,0.4 \Omega_{\mathrm{e}} / 2 \pi(\mathrm{Hz})$ and show evolution of the $\boldsymbol{k}$ vector orientation with respect to $B_{0}$ field.

An interesting feature of these results is as follows. Where the traced rays with different frequencies spread across $L$ shells, particularly after magnetospheric reflection, under the assumption that the entire chorus element is indeed generated at a single point with the same zero propagation angle, then, according to ray tracing theory, a remote observer at a fixed point would only be expected to see a single frequency and not the whole chorus element. This implies that for an observer to see the whole element, one would either have to have ducted propagation or the observer would have to be reasonably close to the generation point so that the rays had not diverged appreciably. However, we note that the real situation is rather more complex, in that the generation region has a finite extent, $\sim 1000$ s of kilometers along the field line, and will radiate into a narrow cone of propagation angles. 
We next compare upper- and lower-band rising frequency chorus, with a starting point at the equator and outside the plasmapause at $L=4.5$. Figure 3 a follows a lower-band rising element with $f=0.3-0.5 \Omega_{\mathrm{e}} / 2 \pi(\mathrm{Hz})$ and Fig. $3 \mathrm{~b}$ follows an upper-band element with $f=0.5-0.7 \Omega_{\mathrm{e}} / 2 \pi(\mathrm{Hz})$. In both cases the wave pulse moves to lower $L$ values, readily crossing the plasmapause, and the propagation angle quickly becomes highly oblique by the time latitude of 10 degrees is reached. It is noticed that the upper-band wave pulses are more field aligned than the lower band, particularly after reflection. Another noticeable feature is that at the highest frequency, $f=0.7 \Omega_{\mathrm{e}} / 2 \pi(\mathrm{Hz})$, the inward propagation of the wave pulse is very marked, having shifted by an amount $\mathrm{d} L \sim-0.3$ by the time 10 degrees of latitude is reached. This would make any nonlinear generation mechanism at these frequencies very difficult and might explain why upperband chorus does not go to high frequencies (Meredith et al., 2012).

The chorus theory of Omura and co-workers (Omura et al., 2008,2009 ) is that the equatorial wave magnetic field amplitude for a rising frequency chorus element is directly related to the frequency sweep rate through the condition that at the equator the inhomogeneity factor $S=-0.4$ for a median value of perpendicular velocity $V_{\perp}$. The rationale for this is as follows. Due to adiabatic effects, growth rates fall off quickly away from the equator, and so we need maximal nonlinear growth at the equator. Nonlinear growth maximizes at $S=-0.4$ for a rising tone, under conditions of constant $S$, and thus we postulate this value of $S$ at the equator. However, since $S$ is a function of perpendicular velocity, we must select this median value of perpendicular velocity $V_{\perp}$ to be that at which the contribution to resonant particle current is maximal. When the zero order distribution function is a bi-Maxwellian with temperature anisotropy $T_{\perp} / T_{\|}=3$, the maximum occurs at a pitch angle in the region of 56 degrees (Nunn et al., 1997; Nunn, 1990). We thus choose $V_{\perp}$ to be $V_{R} \tan \left(66^{\circ}\right)$. In view of the relatively large sweep rates of $+175 \mathrm{kHz} \mathrm{s}^{-1}$ at $L=3.8$, of $+136 \mathrm{kHz} \mathrm{s}^{-1}$ at $L=4.0$ and outside the plasmapause at $L=4.5$ of $+95 \mathrm{kHz} \mathrm{s}^{-1}$, it is of some interest to calculate the corresponding equatorial wave amplitudes. The development proceeds as follows. We define (Omura et al., 2008, 2009)

$\chi^{2}=\frac{1}{1+\xi^{2}}$

and

$\xi^{2}=\frac{\omega\left(\Omega_{\mathrm{e}}-\omega\right)}{\omega_{\mathrm{pe}}^{2}}$,

where $\omega_{\text {pe }}$ is the electron plasma frequency. The wave phase velocity $V_{\mathrm{P}}$ is given by

$V_{\mathrm{P}}=c \chi \xi$

group velocity $V_{\mathrm{G}}$ by (a) Lower band rising tone chorus elements $\left(\omega / \Omega_{e}=0.3-0.5\right)$
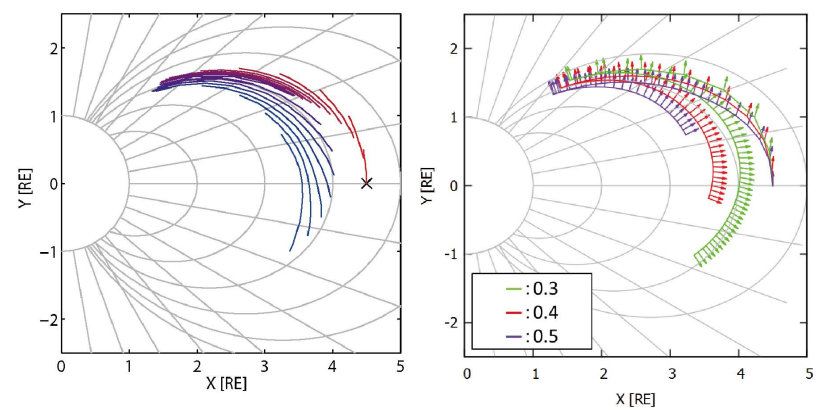

(b) Upper band rising tone chorus elements $\left(\omega / \Omega_{e}=0.5-0.7\right.$ )

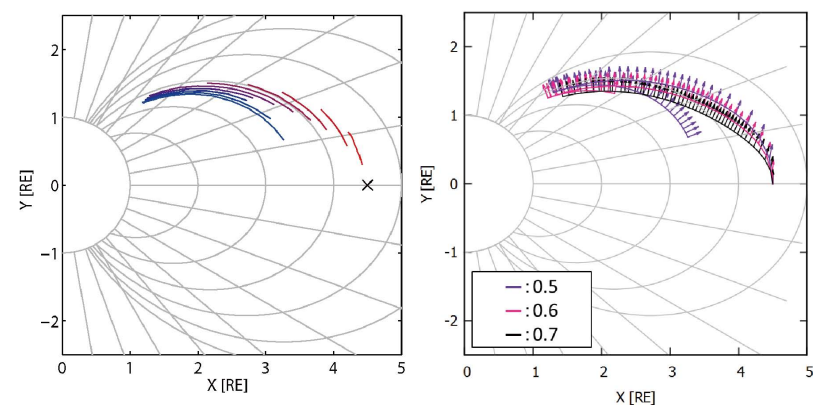

Fig. 3. Ray tracing of rising chorus starting from outside the plasmapause at $L=4.5$ for (a) lower-band chorus element, frequency span $f=0.3-0.5 \Omega_{\mathrm{e}} / 2 \pi(\mathrm{Hz})$ and (b) upper-band chorus element, frequency span $f=0.5-0.7 \Omega_{\mathrm{e}} / 2 \pi(\mathrm{Hz})$. Pulse length is $50 \mathrm{~ms}$ in both cases. Left-hand panel shows pulse evolution, right-hand panel $\boldsymbol{k}$ vector evolution at $f=0.3,0.4,0.5 \Omega_{\mathrm{e}} / 2 \pi(\mathrm{Hz})$ for the lower band and $f=0.5,0.6,0.7 \Omega_{\mathrm{e}} / 2 \pi(\mathrm{Hz})$ for the upper band.

$V_{\mathrm{G}}=\frac{c \xi}{\chi} /\left[\xi^{2}+\frac{\Omega_{\mathrm{e}}}{2\left(\Omega_{\mathrm{e}}-\omega\right)}\right]$,

and cyclotron resonance velocity $V_{\mathrm{R}}$ by

$V_{\mathrm{R}}=c \chi \xi\left(1-\frac{\Omega_{\mathrm{e}}}{\gamma \omega}\right)$.

We next define the coefficients $s_{0}$ and $s_{1}$ as being

$s_{0}=\frac{\chi V_{\perp}}{\xi c}$

and

$s_{1}=\gamma\left[1-V_{\mathrm{R}} / V_{\mathrm{G}}\right]^{2}$,

where $\gamma$ is the relativistic Lorentz factor. Requiring inhomogeneity factor $S=-0.4$ for a value $V_{\perp}$ at the equator gives us the following relation between equatorial wave amplitude and sweep rate:

$\partial f / \partial t=0.4\left(s_{0} / s_{1}\right) f e B_{w} / m$.

Outside the plasmapause at $L=4.5$ for rising chorus at a start frequency of $2.87 \mathrm{kHz}$, we have $B_{w}=395 \mathrm{pT}$. At $L=$ 
4.0 at the plasmapause, this figure becomes $385 \mathrm{pT}$ at a start frequency of $4098 \mathrm{~Hz}$ and inside the plasmapause at $L=3.8$ the figure is also $385 \mathrm{pT}$ at a start frequency of $4779 \mathrm{~Hz}$. These figures correspond to examples of large amplitude chorus outside the plasmapause (Santolik et al., 2003).

We now turn our attention to the case of falling chorus. Figure 4 shows ray tracing for falling tone chorus elements with frequency span $f=0.4-0.2 \Omega_{\mathrm{e}} / 2 \pi(\mathrm{Hz})$, pulse length $50 \mathrm{~ms}$ and pulse separation $50 \mathrm{~ms}$, for (a) $L=3.8$, (b) $L=4.0$, and (c) $L=4.5$. The leading edge of the wave pulse corresponds to the higher frequency $0.40 \Omega_{\mathrm{e}} / 2 \pi(\mathrm{Hz})$. Referring to Nunn and Omura (2012), generating points are located $4000 \mathrm{~km}$ upstream from the equator. Left-hand panels show pulse evolution with time, the right- hand panels $\boldsymbol{k}$ vector evolution for three selected frequencies, $f=0.2,0.3$, $0.4 \Omega_{\mathrm{e}} / 2 \pi(\mathrm{Hz})$. For all $L$ shells and for the whole frequency band, there is considerable obliquity; $\sim 40$ degrees is prevalent by the time the pulse has crossed the equator, with high obliquity of the order of the resonance cone by the time 20 degrees of latitude is reached. For all cases initial pulse shortening occurs across the equator accompanied by $L$ spread, but after reflection considerable pulse lengthening occurs.

In these cases of falling tones, as the localized nose frequency (at least for parallel propagation) is $\sim$ one quarter of the electron gyrofrequency, most of the element is above the nose frequency and thus lower frequencies will travel faster and thus overtake the higher frequencies at the front of the pulse. In the present study initial sweep rates are quite large, and thus here we have "turnover" and a faller becomes a riser. However, by the time that happens the wave train has considerable $L$ shell spread, and it is questionable whether the observer would see the whole chorus element. The inflection in the element wave train profile near the low frequency end corresponds to the nose frequency where group velocity is greatest. Of course the detailed situation is rather complex as rays with different frequencies diverge spatially and group velocity is then a function of local ambient magnetic field, cold plasma density, frequency and propagation angle. A second factor making the higher frequencies slower is that they follow a path with a lower $L$ shell and higher cold plasma density, thus decreasing group velocity still further. In the case of less steep fallers, these propagation effects would steepen the negative frequency gradient but not produce a riser.

We now focus our attention on the region within 10 degrees of latitude of the equator where nonlinear waveparticle interactions can occur. Figure 5 shows the ray tracing for (a) rising chorus and (b) falling chorus. Start points are outside the plasmapause at $L=4.5$, at the equator for the riser and $4000 \mathrm{~km}$ upstream from the equator for the faller. We take an expanded view about the equator.

In the case of the riser, the wave pulse remains reasonably field aligned and only reaches an obliquity of $\sim 30$ degrees at a latitude of 10 degrees. With the faller though, the obliquity is greater than 45 degrees in the whole region downstream
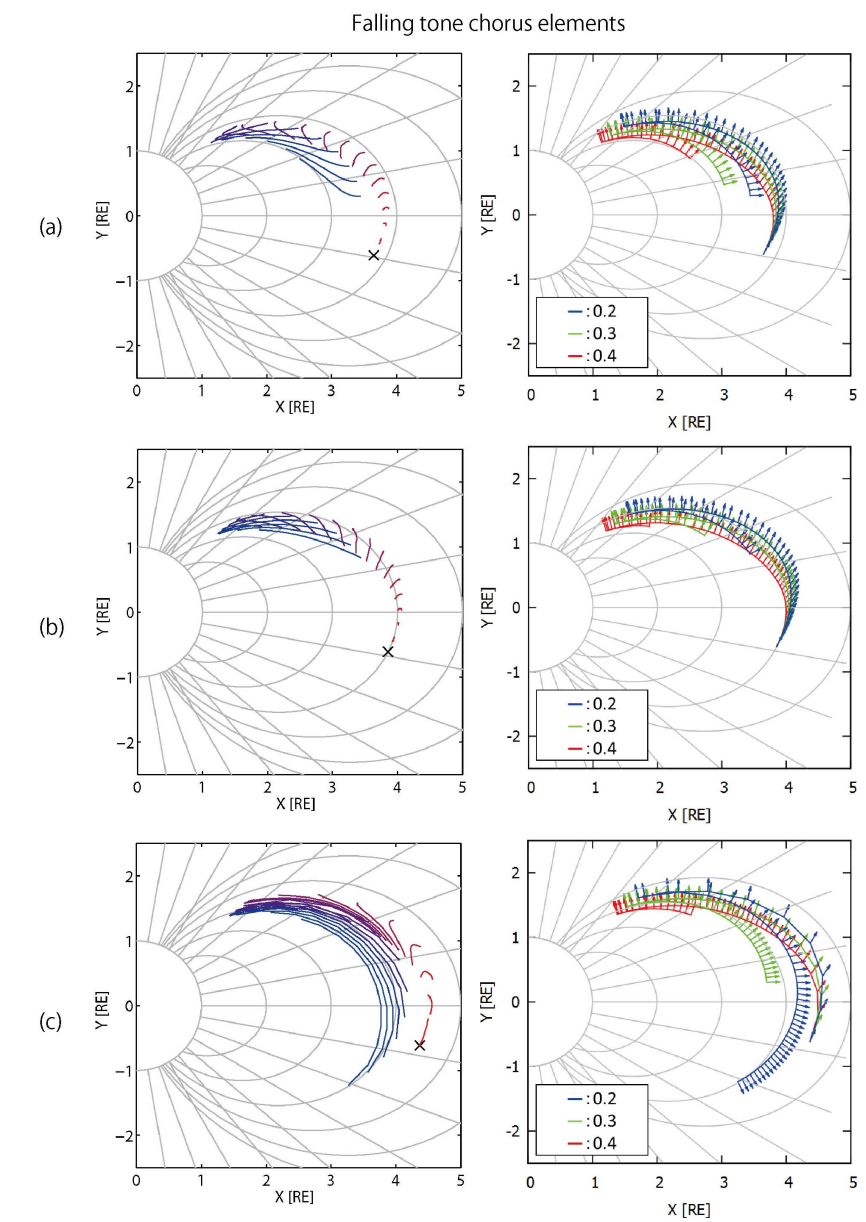

Fig. 4. Ray tracing for falling tone chorus elements with frequency span $f=0.4-0.2 \Omega_{\mathrm{e}} / 2 \pi(\mathrm{Hz})$, pulse length $50 \mathrm{~ms}$ for (a) $L=3.8$, (b) $L=4.0$, and (c) $L=4.5$. In accordance with Nunn and Omura (2012) starting points are $4000 \mathrm{~km}$ upstream from the equator. Left-hand panels show pulse evolution with time, the right-hand panels $\boldsymbol{k}$ vector evolution for three selected frequencies, $f=0.2$, $0.3,0.4 \Omega_{\mathrm{e}} / 2 \pi(\mathrm{Hz})$.

from the equator and there is a significant $L$ spread of the order of $\mathrm{d} L \sim 1 / 4$.

In the literature (Bortnik et al., 2006; Lauben et al., 2002), there are satellite observations to the effect that chorus can be generated in the equatorial region with a propagation angle equal to the Gendrin angle, which is the propagation angle where group velocity is directed along the field line, thus allowing a sufficient interaction length between waves and resonant particles. There are issues of whether the linear and nonlinear growth rates at this propagation angle are sufficient to sustain a generation region. Recently, non-self-consistent computations of nonlinear growth rates in a narrow-band oblique VLF wavefield have been reported (Nunn and Omura, 2012). These computations assume arbitrarily a bi-Maxwellian zero order distribution function, and the wavefield may have any chosen dependence of amplitude, frequency and propagation angle on $z$ and $t$. It was found 
(a) Rising tone $\left(\omega / \Omega_{e}=0.2-0.4\right)$
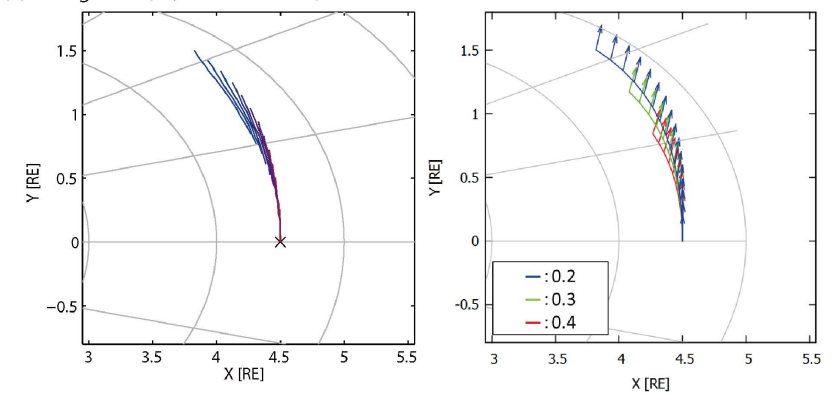

(b) Falling tone $\left(\omega / \Omega_{e}=0.4-0.2\right.$ )
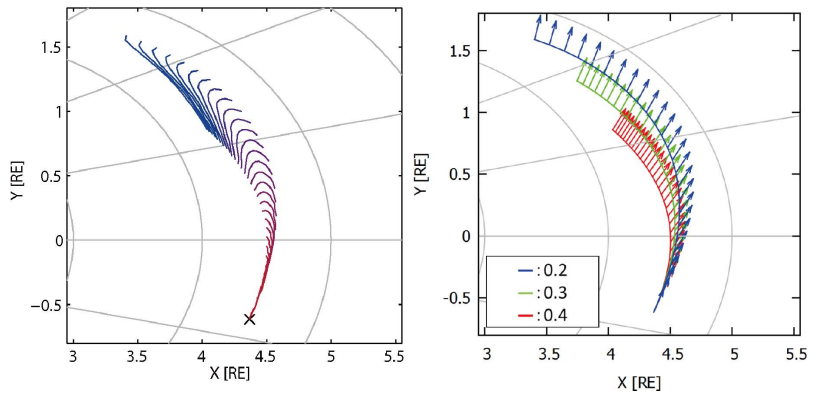

Fig. 5. Expanded equatorial view for $L=4.5$ for lower-band rising and falling tone, $f=0.2-0.4 \Omega_{\mathrm{e}} / 2 \pi(\mathrm{Hz})$ and $f=0.4-0.2 \Omega_{\mathrm{e}} / 2 \pi$ $(\mathrm{Hz})$, respectively. Rising tone is generated at the equator, falling tone $4000 \mathrm{~km}$ upstream from the equator. Left-hand panels show pulse evolution with time; the right-hand panels show $\boldsymbol{k}$ vector evolution for three selected frequencies, $f=0.2,0.3,0.4 \Omega_{\mathrm{e}} / 2 \pi(\mathrm{Hz})$.

that changing the propagation angle from zero to the Gendrin angle reduced the cyclotron growth rate $(n=1$ resonance) by a factor $\sim 4$ from $52 \mathrm{~dB} \mathrm{~s}^{-1}$ to $12 \mathrm{~dB} \mathrm{~s}^{-1}$, and at the same time invoked Landau damping $(n=0$ resonance) of $-6 \mathrm{~dB} \mathrm{~s}^{-1}$. Overall power input was then reduced by a factor of $\sim 8$. Although not ruling out Gendrin angle generation entirely, it would certainly require high particle flux and or high anisotropy. In Fig. 6 we repeat the exercise of Fig. 5 exactly for lower-band chorus, but the rays are all started off at the appropriate Gendrin angle $\psi_{\mathrm{g}}$ for that frequency and location. For the riser the actual values, at the equator, are $\psi_{\mathrm{g}}=\left[\begin{array}{lll}66.4 & 53.0 & 36.8\end{array}\right]$ degrees, corresponding to frequencies $\omega / \Omega_{\mathrm{e}}=\left[\begin{array}{lll}0.2 & 0.3 & 0.4\end{array}\right]$. For the faller the corresponding Gendrin angles are [70.2 59.5 47.4] degrees, all at $h=-4000 \mathrm{~km}$. There is of course the issue of whether in fact in a "Gendrin" chorus generation region propagation angle would track the Gendrin angle as a function of frequency.

Consulting Fig. 6a we see that for the riser the rays remain tightly aligned to the field line within the likely generation region with negligible compression, rotation, expansion or drift of the element wave train. Thus, at least purely from the propagation point of view, a generation region at the Gendrin angle looks possible. The corresponding ray tracing for a faller in Fig. $6 \mathrm{~b}$ is less promising with rotation and inward drift as well as compression of the wave train occurring, making falling chorus at the Gendrin angle look rather unlikely.

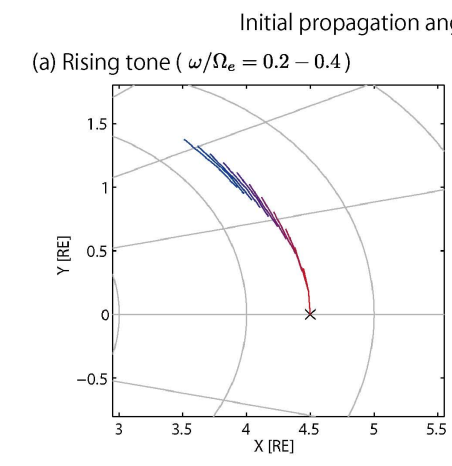

Initial propagation angle at local Gendrin angle
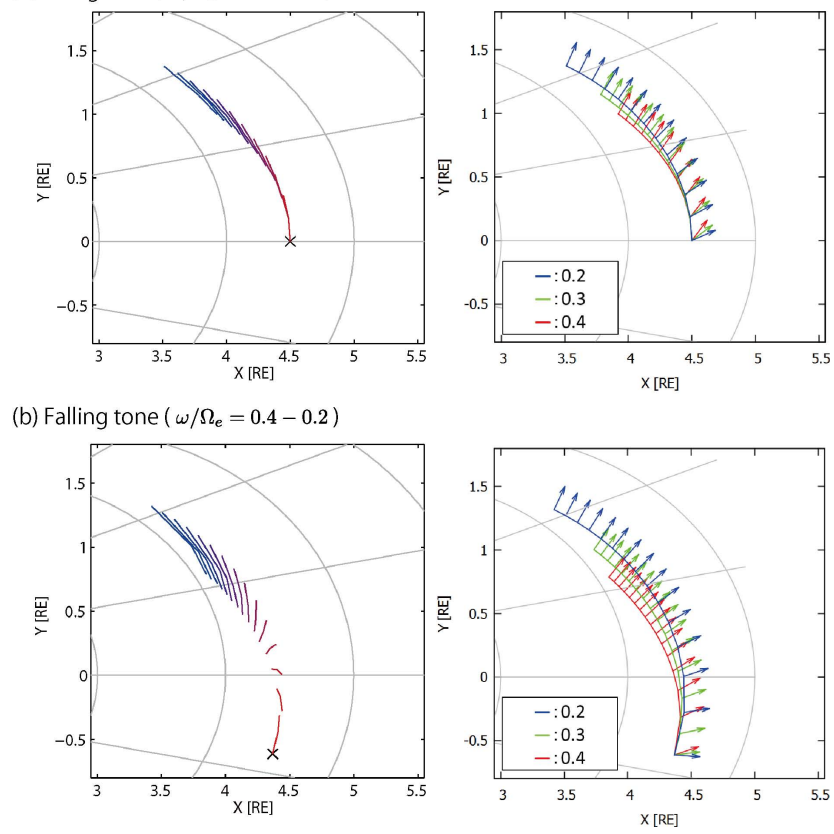

Fig. 6. Expanded equatorial view for $L=4.5$ for lower-band rising and falling tone, $f=0.2-0.4 \Omega_{\mathrm{e}} / 2 \pi(\mathrm{Hz})$ and $f=0.4-0.2 \Omega_{\mathrm{e}} / 2 \pi$ $(\mathrm{Hz})$, respectively, as in Fig. 5, but with all rays starting at the local Gendrin angle.

\section{Discussion and conclusions}

In this paper we have uniquely followed by ray tracing the propagation of the wave pulse of a chorus rising- or fallingfrequency element so as to be able to track its cross $L$ shell motion, as well as the rotation of the wave pulse relative to the field line plus any compression or extension. In addition, the $\boldsymbol{k}$ vector orientation is also determined for sample frequencies within the chorus element bandwidth.

One immediate feature of our results is the tendency for rays to drift inwards in $L$ shell and to readily cross the plasmapause. This is in agreement with calculations in Bortnik et al. (2011), who show that the inward drift of chorus VLF wave energy gives rise to plasmaspheric hiss. Also apparent in general agreement with observations and modelling is the tendency towards oblique propagation at angles approaching the resonance cone once the generation region has been left (Breuillard et al., 2012). There is no sign of quasiparallel propagation once rays recross the equator.

The most significant result of this study is its relevance to the theory of the structure of the generating regions for rising and falling chorus. Following Omura and Nunn (2011) and Nunn and Omura (2012), we place the generating point for a rising element at the equator and for a falling-frequency element upstream of the equator by $4000 \mathrm{~km}$. The rising element remains closely parallel and field aligned within the nonlinear trapping zone out to about 10 degrees of latitude. 
In the case of the faller, the wave pulse remains quasi-parallel and closely field aligned in the nonlinear interaction region upstream from the equator, but downstream from the equator the wavefield becomes oblique $>45$ degrees and spread across $L$ shells. Spreading loss, reduced growth rate due to the $n=1$ cyclotron resonance, Landau damping plus the sharp gradient of propagation angle along the field line will all serve to suppress nonlinear trapping in the downstream region where inhomogeneity factor $S$ is negative. Confining the nonlinear generation region of falling tones to the upstream region with positive inhomogeneity factor $S>0$, as in Nunn and Omura (2012), seems then to have ample justification. Another factor which might have a bearing on cross equatorial coherent wave-particle interaction is azimuthal energetic electron drift. Assuming a drift time of $10 \mathrm{~min}$ around the earth, this gives a drift velocity $\sim 250 \mathrm{~km} \mathrm{~s}^{-1}$, which is not enough to cause particles to be lost from an interaction region of $\sim 100 \mathrm{~km}$ width as estimated in Santolik and Gurnett (2003).

A rigorous treatment of the problem will require a full 3-D simulation in which propagation and nonlinear wave-particle interaction are dealt with in a unified code, but this remains just beyond current computer capabilities. It is not impossible that the nonlinear component of resonant particle current $J_{b}$ might act to self-focus the wavefield along the field line, but this seems unlikely and must remain a topic for further research.

Finally, it should be pointed out that our DE cold plasma density model is smooth. It is quite likely that plasma density in the plane perpendicular to $B_{0}$ may have variations and irregularities which can act to "duct" the wavefield along the field line. If some kind of ducting is occurring, then the conclusions from this paper will not be valid. In this case cross-equatorial coherent wave-particle interaction occurs. The trapping initially takes place in the downstream $S<0$ region producing a distribution function hole. This will propagate across the equator and delay or suppress the formation of a distribution function hill, and thus prevent formation of a positive $J_{b}$ upstream, thus making a faller generation region difficult or impossible. It is quite possible that such ducting does sometimes occur which might explain the overall tendency towards rising chorus.

Acknowledgements. One author, DN, gratefully acknowledges Kyoto University for a Visiting Professorship in 2012. All authors acknowledge the use of the ray tracing code of I. Kimura. This work was supported by Grant-in-Aid 23340147 of the Ministry of Education, Culture, Sports, Science and Technology in Japan.

Topical Editor R. Nakamura thanks two anonymous referees for their help in evaluating this paper.

\section{References}

Bortnik, J., Inan, U. S., and Bell, T. F.: Landau damping and resultant unidirectional propagation of chorus waves, Geophys. Res. Lett., 33, L03102, doi:10.1029/2005GL024553, 2006.

Bortnik, J., Chen, L., Li, W., Thorne, R. M., Meredith, N. P., and Horne, R. B.: Modeling the wave power distribution and characteristics of plasmaspheric hiss, J. Geophys. Res., 116, A12209, doi:10.1029/2011JA016862, 2011.

Breuillard, H., Zaliznyak, Y., Krasnoselskikh, V., Agapitov, O., Artemyev, A., and Rolland, G.: Chorus wave-normal statistics in the Earth's radiation belts from ray tracing technique, Ann. Geophys., 30, 1223-1233, doi:10.5194/angeo-30-1223-2012, 2012.

Chen, L., Bortnik, J., Thorne, R. M., Horne, R. B., and Jordanova, V. K.: Three dimensional ray tracing of VLF waves in a magnetospheric environment containing a plasmaspheric plume, Geophys. Res. Lett., 36, L22101, doi:10.1029/2009GL040451, 2009.

Haselgrove, J.: Ray theory and a new method for ray tracing, Report of Conference on the Physics of the Ionosphere, London Physical Society, 355-364, 1954.

Hayakawa, M., Yamanaka, Y., Parrot, M., and Lefeuvre, F.: The wave normals of magnetospheric chorus emissions observed on board GEOS 2, J. Geophys. Res., 89, 2811-2821, doi:10.1029/JA089iA05p02811, 1984.

Hiroyasu, M. and Masashi, M.: Ray-tracing study of the propagation in the magnetosphere of whistler-mode VLF emissions with frequency above half the electron gyrofrequency, Planet. Space Sci., 35, 1397-1404, 1987.

Hikishima, M., Yagitani, S., Omura, Y., and Nagano, I.: Full particle simulation of whistler-mode rising chorus emissions in the magnetosphere, J. Geophys. Res., 114, A01203, doi:10.1029/2008JA013625, 2009.

Hikishima, M., Omura, Y., and Summers, D.: Self-consistent particle simulation of whistler-mode triggered emissions, J. Geophys. Res., 115, A12246, doi:10.1029/2010JA015860, 2010a.

Hikishima, M., Omura, Y., and Summers, D.: Microburst precipitation of energetic electrons associated with chorus wave generation, Geophys. Res. Lett., 37, L07103, doi:10.1029/2010GL042678, 2010b.

Horne, R. B.: Path-integrated growth of electrostatic waves: The generation of terrestrial myriametric radiation, J. Geophys. Res., 94, 8895-8909, 1989.

Horne, R. B. and Thorne, R. M.: Relativistic electron acceleration and precipitation during resonant interactions with whistler-mode chorus, Geophys. Res. Lett., 30, 1527, doi:10.1029/2003GL016973, 2003.

Katoh, Y. and Omura, Y.: Computer simulation of chorus wave generation in the Earth's inner magnetosphere, Geophys. Res. Lett., 34, L03102, doi:10.1029/2006GL028594, 2007a.

Katoh, Y. and Omura, Y.: Relativistic particle acceleration in the process of whistler-mode chorus wave generation, Geophys. Res. Lett., 34, L13102, doi:10.1029/2007GL029758, 2007b.

Kimura, I.: Effects of ions on whistler-mode ray tracing, Radio Science, 1, 269-283, 1966.

Kimura, I. and Goto, Y.: Ray Tracing, available at: http://waves.is. t.kanazawa-u.ac.jp/index.php?Ray (last access: 10 April 2013), 2010.

Kurita, S., Katoh, Y., Omura, Y., Angelopoulos, V., Cully, C. M., Le Contel, O., and Misawa, H.: THEMIS observation of chorus elements without a gap at half the gyrofrequency, J. Geophys. 
Res., 117, A11223, doi:10.1029/2012JA018076, 2012.

Lauben, D. S., Inan, U. S., Bell, T. F., and Gurnett, D. A.: Source characteristics of VLF chorus, J. Geophys. Res., 107, 1429, doi:10.1029/2000JA003019, 2002.

Li, W., Thorne, R. M., Bortnik, J., Shprits, Y. Y., Nishimura, Y., Angelopoulos, V., Chaston, C., Le Contel, O., and Bonnell, J. W.: Typical properties of rising and falling tone chorus waves, Geophys. Res. Lett., 38, L14103, doi:10.1029/2011GL047925, 2011.

Meredith, N. P., Horne, R. B., Sicard-Piet, A., Boscher, D., Yearby, K. H., Li, W., and Thorne, R. M.: Global model of lower band and upper band chorus from multiple satellite observations, J. Geophys. Res., 117, A10225, doi:10.1029/2012JA017978, 2012.

Nunn, D.: The Numerical Simulation of Non Linear VLF Wave Particle Interactions Using the Vlasov Hybrid Simulation Technique, Comput. Phys. Commun., 60, 1-25, 1990.

Nunn, D. and Omura, Y.: A computational and theoretical analysis of falling frequency VLF emissions, J. Geophys. Res., 117, A08228, doi:10.1029/2012JA017557, 2012.

Nunn, D., Omura, Y., Matsumoto, H., Nagano, I., and Yagitani, S.: The numerical simulation of VLF chorus and discrete emissions observed on the Geotail satellite, J. Geophys. Res., 102, 2708327097, 1997.

Nunn, D., Santolik, O., Rycroft, M., and Trakhtengerts, V.: On the numerical modelling of VLF chorus dynamical spectra, Ann. Geophys., 27, 2341-2359, doi:10.5194/angeo-27-23412009, 2009.

Omura, Y. and Nunn, D.: Triggering process of whistler mode chorus emissions in the magnetosphere, J. Geophys. Res., 116, A05205, doi:10.1029/2010JA016280, 2011.

Omura, Y., Katoh, Y., and Summers, D.: Theory and simulation of the generation of whistler-mode chorus, J. Geophys. Res., 113, A04223, doi:10.1029/2007JA012622, 2008.
Omura, Y., Hikishima, M., Katoh, Y., Summers, D., and Yagitani, S.: Nonlinear mechanisms of lower-band and upper-band VLF chorus emissions in the magnetosphere, J. Geophys. Res., 114, A07217, doi:10.1029/2009JA014206, 2009.

Persoon, A. M., Gurnett, D. A., Santolik, O., Kurth, W. S., Faden, J. B., Groene, J. B., Lewis, G. R., Coates, A. J., Wilson, R. J., Tokar, R. L., Wahlund, J.-E., and Moncuquet, M.: A diffusive equilibrium model for the plasma density in Saturn's magnetosphere, J. Geophys. Res., 114, A04211, doi:10.1029/2008JA013912, 2009.

Pickett, J. S., Santolík, O., Kahler, S. W., Masson, A., Adrian, M. L., Gurnett, D. A., Bell, T. F., Laakso, H., Parrot, M., Décréau, P., Fazakerley, A., Cornilleau-Wehrlin, N., Balogh, A., and André, M.: Multi-point CLUSTER observations of VLF risers, fallers and hooks at or near the plasmapause, edited by: Sauvaud, J.-A., Multiscale Processes in the Earth's Magnetosphere: from Interball to Cluster, 307-328, Kluwer Academic Publishers, Holland, 2004.

Richardson, J. D. and Sittler Jr., E. C.: Laboratory f or a plasma density model for Saturn based on Voyager observations, J. Geophys. Res., 95, 12019-12031, 1990.

Santolik, O. and Gurnett, D. A.: Transverse dimensions of chorus in the source region, Geophys. Res. Lett., 30, 1031, doi:10.1029/2002GL016178, 2003.

Santolik, O., Gurnett, D. A., and Pickett, J. S.: Spatio-temporal structure of storm time chorus, J. Geophys. Res., 108, 1278, doi:10.1029/2002JA009791, 2003.

Santolik, O., Gurnett, D. A., Pickett, J. S., Chum, J., and CornilleauWehrlin, N.: Oblique propagation of whistler mode waves in the chorus source region, J. Geophys. Res., 114, A00F03, doi:10.1029/2009JA014586, 2009.

Stix, T. H.: Waves in Plasmas, American Institute of Physics, 1992. 